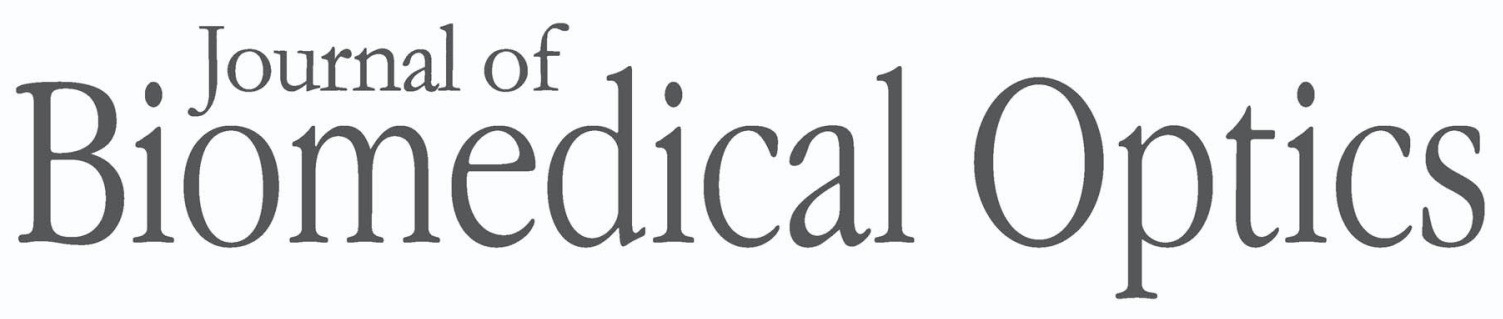

\title{
Role of the prefrontal cortex in the cognitive control of reaching movements: near-infrared spectroscopy study
}

Kotaro Goto

Yoko Hoshi

Masashi Sata

Masatoshi Kawahara

Makoto Takahashi

Harumitsu Murohashi 


\title{
Role of the prefrontal cortex in the cognitive control of reaching movements: near-infrared spectroscopy study
}

\author{
Kotaro Goto, ${ }^{a, *}$ Yoko Hoshi, ${ }^{b}$ Masashi Sata, ${ }^{c}$ Masatoshi Kawahara, ${ }^{\mathrm{c}}$ Makoto Takahashi, ${ }^{\mathrm{c}}$ and Harumitsu Murohashi ${ }^{\mathrm{a}}$ \\ ${ }^{a}$ Hokkaido University, Graduate School of Education, Sapporo, Japan \\ ${ }^{b}$ Tokyo Metropolitan Institute of Medical Science, Integrated Neuroscience Research Project, 2-1-6 Kamikitazawa, \\ Setagaya-ku, Tokyo 156-8503 Japan \\ ${ }^{\mathrm{c}}$ Hokkaido University, Graduate School of Information Science and Technology, Sapporo, Japan
}

\begin{abstract}
To elucidate the role of the prefrontal cortex in cognitive control of reaching movements, by multichannel near-infrared spectroscopy we examine changes in oxygenated hemoglobin (oxy-Hb) as an indicator of changes in regional cerebral blood flow in the bilateral dorsolateral (DLPFC), ventrolateral prefrontal cortex (VLPFC), and frontopolar cortex (FPC) during a reaching task with normal visual feedback (a consistent task) and a reaching task with flipped horizontal visual feedback (an inconsistent task). Subjects first perform 12 trials of the consistent task, and then perform six blocks of the inconsistent task, each of which consists of six trials. During the consistent task, $\mathrm{oxy}-\mathrm{Hb}$ is increased only in the right VLPFC. During the first block of the inconsistent task, increases in oxy- $\mathrm{Hb}$ are observed in the bilateral DLPFC and the right VLPFC, whereas the increased oxy- $\mathrm{Hb}$ was gradually reduced as the block proceeded, which was accompanied by an improvement in the task performance. Eventually, there were no differences in the degree of change in oxy-Hb between the consistent and inconsistent tasks in the DLPFC and VLPFC. These findings suggest that the DLPFC is engaged in higher order cognitive control, while the right VLPFC is engaged in both higher and lower order cognitive controls. $@ 2011$ Society of Photo-Optical Instrumentation Engineers (SPIE). [DOI: 10.1117/1.3658757]
\end{abstract}

Keywords: near-infrared spectroscopy; reaching; visuomotor transformations; ventrolateral prefrontal cortex; dorsolateral prefrontal cortex; frontopolar cortex; oxy-Hb.

Paper 11403R received Jul. 26, 2011; revised manuscript received Oct. 10, 2011; accepted for publication Oct. 18, 2011; published online Nov. 28, 2011.

\section{Introduction}

The task of reaching for something, although simple and automatic, actually involves complex neural mechanisms; the motor output is guided by integration of visual and proprioceptive feedback. ${ }^{1,2}$ Reaching movements, which are defined as fast ballistic voluntary movements toward a given target, have been used as approaches to elucidate mechanisms of motor control and learning in computational neuroscience, ${ }^{1,3}$ however, little attention has been paid to the prefrontal cortex (PFC), at the summit of the perception-action cycle. ${ }^{4}$ The role of the PFC in motor behavior, which is not restricted to reaching movements, has been investigated in primates by using electrophysiological methods. ${ }^{5-7}$ These studies have shown that the dorsolateral prefrontal cortex (DLPFC) is engaged in a range of aspects of the cognitive control of motor behavior, such as processing sensory inputs and planning of future actions.

Neural mechanisms corresponding to human reaching movements have been investigated by using neuroimaging techniques. Studies of human adults with positron emission tomography (PET) and functional magnetic resonance imaging (fMRI) have demonstrated that reaching tasks are accompanied by activation of the motor cortex, somatosensory cortex, basal ganglia, cerebellum, premotor cortex, supplementary motor area, cingulate

*Present address: Tokyo University of Agriculture, Faculty of Bioindustry, Abashiri, Japan.

Corresponding address: Yoko Hoshi, Tokyo Metropolitan Institute of Medical Science, Integrated Neuroscience Research Project, 2-1-6 Kamikitazawa, Setagayaku, Tokyo 156-8503 Japan; Tel: 81-3-6834-2319; Fax: 81-3-6834-2319, E-mail: hoshi-yk@igakuken.or.jp. cortex, and parietal cortex. ${ }^{8-10}$ However, only a few studies have reported that the PFC is activated during reaching tasks. ${ }^{10,11}$ Fuster $^{4}$ has argued that the PFC plays a critical role in the meditation of contingencies of action across time based on the interplay of sensory and motor working memories; whereas, when a behavior has become automatic, it is not involved in the processing of action. This is consistent with observations from neuroimaging and electrophysiological studies where the PFC, especially the DLPFC, was activated during learning of a new motor behavior and the degree of activation declined as learning progressed. ${ }^{13}$ Because the majority of daily human motor behaviors, including reaching movements, are well acquired during childhood, it may be presumed that the PFC is rarely engaged in reaching movements in adults. This can account for the fact that only a few neuroimaging studies have reported PFC activation during reaching tasks.

Relationships between visual and proprioceptive information can be transformed within behavioral settings using prism lenses,${ }^{14}$ computer-generated tasks, ${ }^{15}$ and reversing spectacles. ${ }^{16}$ It is presumed that reaching tasks under these circumstances are accompanied by activation of the PFC because adaptation to transformed visuomotor relationships involves executive functions, such as inhibition and working memory. The neural correlates of sensorimotor adaptation have been investigated from various viewpoints, such as sensorimotor plasticity and motor learning. ${ }^{17-19}$ Contrary to our expectations, most of these studies have proposed the importance of the cerebellum and the parietal cortex for sensorimotor adaptation. Too little

1083-3668/2011/16(12)/127003/8/\$25.00 @ 2011 SPIE 
has been reported on the involvement of the PFC. ${ }^{20,21}$ Overall, the findings thus far suggest that the PFC may be engaged only in the early stage of sensorimotor adaptation like other forms of motor learning.

The PFC is cytoarchitectonically and functionally considered a heterogeneous region, and in monkeys, the ventral and dorsal regions of the lateral PFC play different roles in cognitive control of motor behavior. ${ }^{22}$ In humans, the role of each subregion of the PFC in reaching movements remains to be fully understood. PET and fMRI have contributed to elucidating aspects of the functional anatomy of the human brain; however, there are technical difficulties in applying these techniques to reaching movement studies. For example, the range of reaching tasks that can be performed in a PET and/or fMRI measurement environment are limited. Unlike PET and fMRI, near-infrared spectroscopy (NIRS) enables continuous measurements of brain activity-related changes in cerebral hemoglobin $(\mathrm{Hb})$ in real time, in everyday environments, ${ }^{23-25}$ making it an attractive option for investigating the role of the PFC during reaching movements. Thus far, only a few NIRS studies on reaching movements have been reported where the frontopolar cortex (FPC) and the dorsal PFC have been examined, ${ }^{26-28}$ but not the lateral PFC.

In this study, the role(s) of three subregions of the PFC [the FPC, the ventrolateral prefrontal cortex (VLPFC), and the DLPFC] in cognitive control of reaching movements were examined. A multichannel NIRS instrument was employed to measure cerebral $\mathrm{Hb}$ changes in the three subregions of the PFC during two reaching tasks: $(i)$ a reaching task with a normal visual feedback (a consistent task) and (ii) a reaching task with inconsistent visual and proprioceptive feedback (an inconsistent task). Because adults can automatically perform reaching movements with normal visual feedback, it was hypothesized that the PFC, especially the DLPFC, would not be activated during the consistent task. It was further hypothesized that the PFC was engaged only in the early stage of adaptation of reaching movements to visuomotor transformations.

\section{Methods}

For this study, the PFC was divided into the DLPFC, VLPFC, and FPC. The anatomical extent of the FPC is not generally agreed on; however, in this study it is defined as the lateral portion of Brodmann area 10 (BA 10) and the interim areas on the lateral borderline of BA $10 .^{29}$ The bilateral DLPFC (BAs 9/46) and VLPFC (BAs 45/47) were examined in experiment 1 , and the FPC in experiment 2.

\subsection{Experiment 1}

\subsubsection{Subjects}

Ten right-handed healthy adults (four males and six females; ages from 19 to 29 years, mean age 24.4 years) participated in the study. None had a history of neurological illness, head injury, or substance abuse. Written informed consent was obtained from all the subjects. The experiments were performed according to the policies and principles in the Declaration of Helsinki.

\subsubsection{Apparatus for reaching tasks}

Figure 1(a) shows the scheme of the experimental apparatus for reaching tasks. Subjects sat in front of a table and attached a (a)

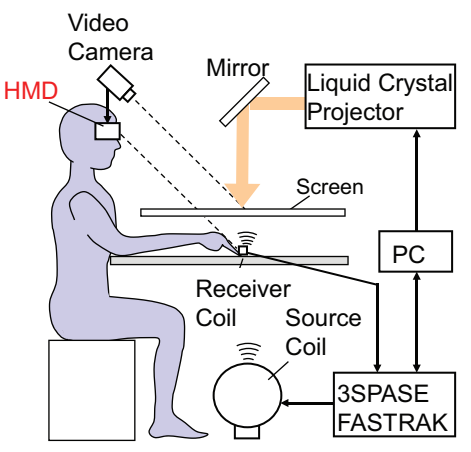

(b)

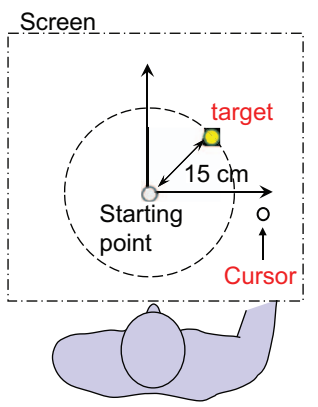

Fig. 1 (a) Scheme of the experimental apparatus for reaching tasks and (b) virtual images of on the screen.

receiver coil to the tip of the right index finger. The subjects had a head-mounted display (HMD) (PLM-S700, Sony, Japan) attached to their heads, and the head was restrained by a chin rest. When performing reaching movements on the table, the position of the receiver coil was followed by a three-dimensional magnetic sensor (3SPACE FASTRAK, Polhemus, Vermont) at a sampling rate of $100 \mathrm{~Hz}$ and a spatial resolution of $0.8 \mathrm{~mm}$. The position of the cursor was calculated every $0.01 \mathrm{~s}$ with software developed in house. A target and a cursor that represented the position of the index finger position were displayed in realtime as virtual images on a screen that was placed $20 \mathrm{~cm}$ above the table by using a liquid-crystal projector (ELP-3500, Epson, Japan) [Fig. 1(b)]. The diameters of the cursor, the starting point, and the target were 20,16 , and $16 \mathrm{~mm}$, respectively. A screen that prevented the subjects from directly viewing their fingers was captured by a video camera (CCD-MC100, Sony, Japan), and the visual images on the screen were also simultaneously displayed on the HMD. The starting point and cursor were illuminated throughout the duration of the experiment. In a few seconds (1-2 s) after the subjects put the cursor on the starting point, the target appeared and remained there until the cursor reached and overlapped it. After reaching the target, the subjects were instructed to move the cursor back toward the starting point.

\subsubsection{Experimental design}

Two reaching tasks were investigated: $(i)$ a reaching task with normal visual feedback (a consistent task) and (ii) a reaching task with inconsistent visual and proprioceptive feedback (an inconsistent task) (Fig. 2). In both the tasks, the target was located at a distance of $15 \mathrm{~cm}$ from the starting position on the right side at a 45-deg angle to the sagittal midline of the trunk of the subject. Because in the inconsistent task the cursor did not represent the right index finger position, but a left-right reversed position, the subjects had to deal with conflicting visual and proprioceptive information by combining the information into an estimate of their actual index finger position. Thus, higher order cognitive control and learning, which is hereafter referred to as visuomotor transformation learning ${ }^{18}$ were required to perform the inconsistent task. After a 1 min resting period, each subject first performed 12 trials of the consistent task (the first block) and then performed six blocks of the inconsistent task, each of which consisted of six successive trials. The last block 
(a) consistent task

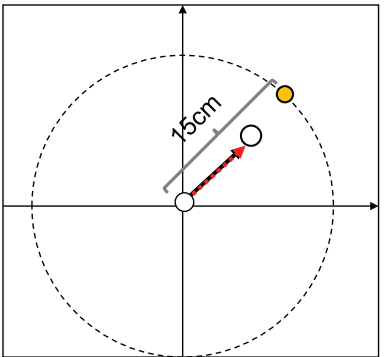

(b) inconsistent task

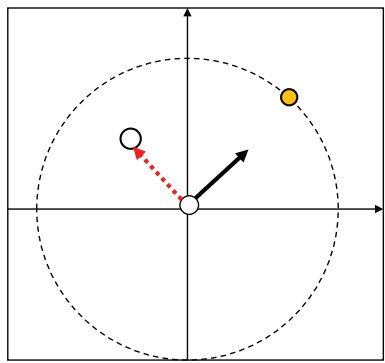

Fig. 2 Schematic illustration of the (a) consistent and (b) inconsistent reaching task. The solid and dotted arrows denote the finger and cursor path, respectively. In the inconsistent task, the cursor is displayed at its left-right reversed position.

(the seventh block) was followed by a last resting period. The interblock interval of 1 min was regarded as a resting period for the following block of tasks. A normal reaching is, in general, well acquired. However, in our previous study, we found that some subjects got nervous and did not perform the first few trials (one or two trials) of the consistent task smoothly. To avoid these data from influencing statistical results, the number of trials for the consistent task was determined to be 12 . The subjects were instructed to reach the target using their dominant hand (right hand) as quickly and accurately as possible soon after the target appeared, and to move the cursor back toward the starting point after reaching the target. The subjects were also instructed to look at a white cross hair presented at the center of the screen during resting periods until the target appeared, and were informed that visual information about the finger position would not necessarily agree with proprioceptive information in some blocks. The receiver coil was attached to each subject's finger tip $10 \mathrm{~min}$ prior to the measurement, but no practice was permitted.

\subsubsection{Task performance}

Task performance was evaluated by three behavioral measures: trajectory error (TE), initial movement direction (IMD), and movement time (MT). The TE was calculated by subtracting the start-to-target distance in a straight line from the length of the trajectory traced by the movement. The IMD was expressed by an angle with the start-to-target line as the base, at $0 \mathrm{deg}$ (Fig. 3). The MT was defined as the duration of the movement from the starting position to the target, which was calculated by

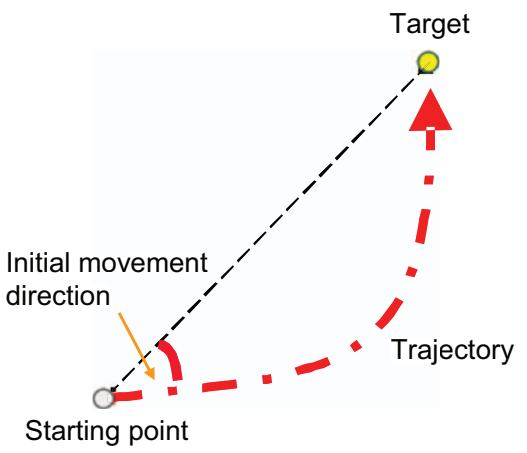

Fig. 3 Initial movement direction and trajectory. (a)

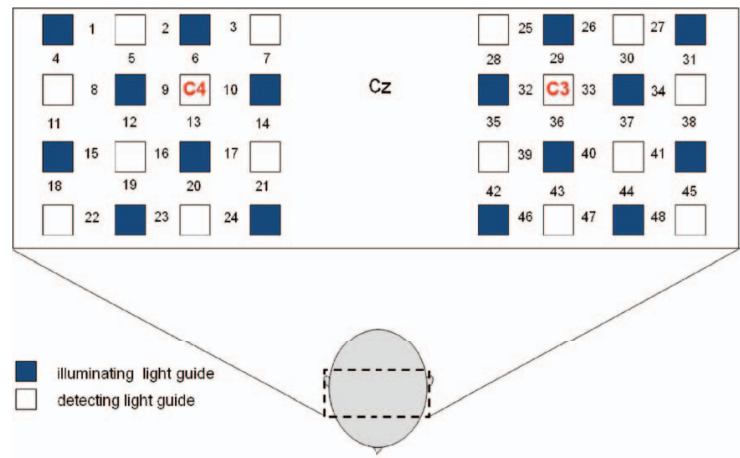

(b)

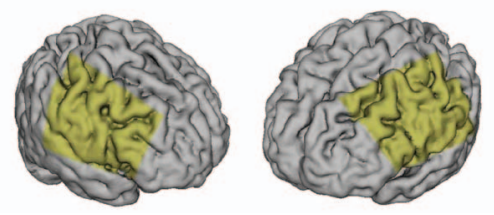

Fig. 4 (a) Positions of light guides in experiment 1. Hb changes were measured at 48 regions between each pair of source and detector. We termed these regions as channels (ch) and tentatively decided their nomenclature, as shown in the figure. (b) The brain area beneath the light guides for one subject is shown by a shaded square. In this subject, channels 16-19-23 (right VLPFC), 40-44-47 (left VLPFC), 17-21-24 (right DLPFC) and 39-43-46 (left DLPFC), were selected.

using a timer function. The task performance for each block of trials was expressed as an average of task performance on each trial in one block.

\subsubsection{Near-infrared spectroscopy and data analysis}

A multichannel NIRS imaging system (OMM-3000, Shimadzu Company, Kyoto, Japan) was employed to measure $\mathrm{Hb}$ changes related to brain activity. The NIRS system used three wavelength semiconductor laser diodes (780, 805, and $830 \mathrm{~nm})$ and calculated changes in the $\mathrm{Hb}$ based on the modified Beer-Lambert law. ${ }^{30}$ Because the NIRS apparatus of this type does not provide absolute values of $\mathrm{Hb}$ concentration changes, $\mathrm{Hb}$ changes are expressed by arbitrary units. ${ }^{31}$ The NIRS signals were sampled every $145 \mathrm{~ms}$. The illuminating and detecting light guides, which were bundle fibers (2-mm diam), were symmetrically placed on the lateral frontal region (an area of $9 \times 9 \mathrm{~cm}$ ) of both sides with a source-detector separation of $3 \mathrm{~cm}$ by using $\mathrm{C} 3$ and $\mathrm{C} 4$ of the international 10-20 system for electroencephalography (EEG) electrode placement as landmarks, and a total of 48 regions (channels) were measured [Figs. 4(a) and 4(b)]. The NIRS measurements were continuously performed from 2 or 3 min before the first resting period to the end of the last resting period (total duration, about $20 \mathrm{~min}$.). On completion of the study, the subjects underwent MRI measurement to confirm the brain regions beneath each light guide. An electromagnetic tracking device (FASTRAK, Polhemus, USA) was used to digitize light guide positions. Digitized light guide positions were marked on the head surface and registered to 3-D surface rendered MR images of the brain.

Because the head shape and size were different for each subject, channels corresponding to four regions of interest (ROIs) the bilateral DLPFC (BAs 9/46, middle frontal gyrus) and VLPFC (BAs 45/47, inferior frontal gyrus) — were individually identified based on the MR images for a subject. All channels 
within each ROI were selected for further analysis. In the present study, oxy-Hb, which is the most sensitive indicator of changes in $\mathrm{CBF}^{32}$ was analyzed. For a given subject and channel, taking the value of oxy-Hb at the beginning of the first trial in each block of tests as 0 (the baseline), changes in oxy-Hb were recalculated for every block. Averaged "trial" oxy-Hb values were calculated by averaging all data during the test block period. Averaged "first-resting" oxy-Hb values were calculated by averaging $30 \mathrm{~s}$ of data, prior to the start of the consistent task (the first block) in the same manner. These values were averaged across all selected channels.

\subsubsection{Statistical analysis}

The averaged task performance and oxy-Hb change in each block for all the subjects were analyzed by using a one-way repeated measures analysis of variance (ANOVA). Then, a paired $\mathrm{t}$-test was performed on comparisons of the task performance and changes in oxy-Hb between the consistent task and the first block of the inconsistent task, the first and last blocks of the inconsistent task, and the consistent task and the last block of the inconsistent task. A paired t-test was also used to compare changes in oxy-Hb between the first resting period and the consistent task. In the present study, $P<0.01$ (Bonferroni correction, 0.05/4) was chosen as the level of significance. The relationship between changes in oxy-Hb and task performances was examined by Pearson product-moment correlation coefficients.

\subsection{Experiment 2}

Except for measured brain regions and subjects, the method of experiment 2 was the same as that of experiment 1 . To avoid the effect of adaptation to the inconsistent visual and proprioceptive feedback, experiment 2 was performed by subjects who did not participate in experiment 1 .

\subsubsection{Subjects}

Twelve right-handed healthy adults (six males and six females; ages from 19 to 30 years, mean age 24.6 years) participated in this study. None had a history of neurological illness, head injury, or substance abuse. Written informed consent was obtained from all the subjects. The experiments were performed according to the policies and principles in the Declaration of Helsinki.

\subsubsection{Near-infrared spectroscopy and data analysis}

Fourteen illuminating and detecting light guides were placed on the anterior frontal region (an area of $9 \times 18 \mathrm{~cm}$ ) with a sourcedetector separation of $3 \mathrm{~cm}$ by using the Fpz of the international 10-20 system for EEG electrode placement as a landmark [Figs. 5(a) and 5(b)]. According to MRI measurements, it was confirmed that the measured brain area included the FPC in all the subjects. Patterns of changes in oxy-Hb in the channels included in the FPC were similar within an individual subject. Compared to the VLPFC and DLPFC measurement (experiment 1), intersubject variations of the channel locations were relatively small. For further analyses, thus, channels 28-34-35-41 and 31-37-3844, which measured the right and left FPC, respectively, were selected. (a)

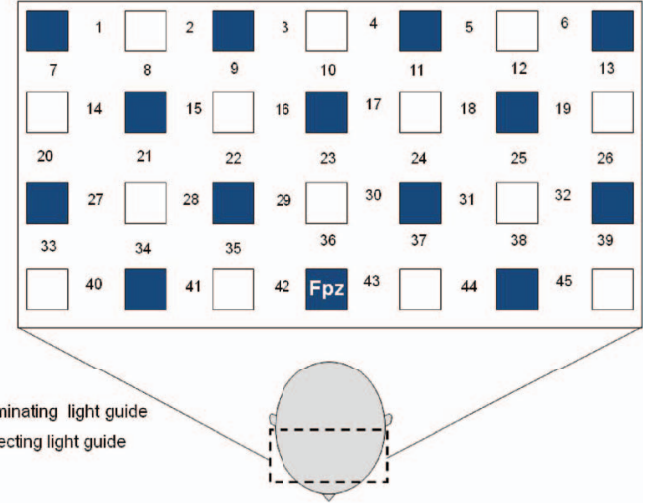

(b)

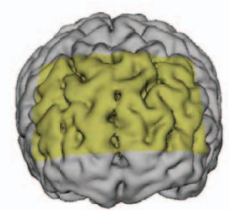

Fig. 5 (a) Positions of light guides in experiment 2. Hb changes were measured at 45 regions between each pair of source and detector. (b) The brain area beneath the light guides for a subject different from one in Fig. 4(b) is shown by a shaded square.

\section{Results}

\subsection{Task performance}

Because there were no differences in all the three behavioral measures between experiments 1 and 2 (TE, $F(1)=0.24$, $P=0.625 ; \mathrm{IMD}, F(1)=1.89, P=0.172 ; \mathrm{MT}, F(1)$ $=0.931, P=0.352$ ), we combined data of both experiments. Figures 6(a) and 6(c)- show the TE, IMD, and MT during the consistent and inconsistent tasks, respectively. There were significant main effects of the block in task performances between blocks [TE, $F(1.26)=36.35, P<0.001$; IMD, $F(3.11)=23.37$, $P<0.001$; MT, $F(1.38)=27.65, P<0.001]$. During the consistent task, the TE, IMD, and MT were $19.14 \pm 13.10 \mathrm{~mm}$ (mean $\pm \mathrm{SD}$ ), $6.20 \pm 1.55 \mathrm{deg}$ and $1.68 \pm 0.11 \mathrm{~s}$, respectively. Compared to the consistent task, the task performance was much poorer during the first block of the inconsistent task $[\mathrm{TE}, t(21)=6.15, p<0.001 ; \mathrm{IMD}, t(21)=9.18, p<0.001$; MT, $t(21)=7.0, p<0.001]$. However, the task performance was gradually improved with block repetitions, and it became much better in the last block than it had been in the first block of the inconsistent task (TE, $t(21)=6.11, p<0.001$; IMD, $t(21)$ $=9.84, p<0.001$; MT, $t(21)=7.01, p<0.001)$. And there were no significant differences in the TE, IMD, and MT between the last block of the inconsistent task and the consistent task.

\subsection{Changes in brain activity}

\subsubsection{DLPFC}

Figure 7 shows the changes in the oxy-Hb in each block in the bilateral DLPFC for all the subjects. Significant main effects of the block in oxy-Hb changes between task blocks were observed in both sides of the DLPFC [left, $F(2.76)=15.0, P<0.001$; right, $F(2.03)=9.13, P<0.005$ ]. Compared to the first resting period, the consistent task was not accompanied by significant increases in oxy-Hb in the bilateral DLPFC. By contrast, in 
(a)

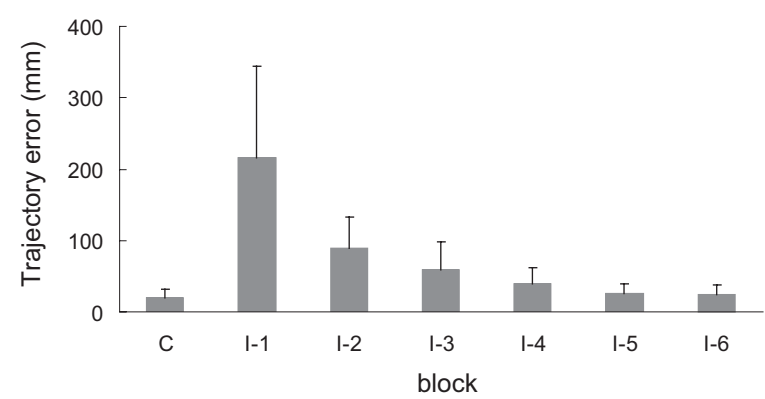

(b)

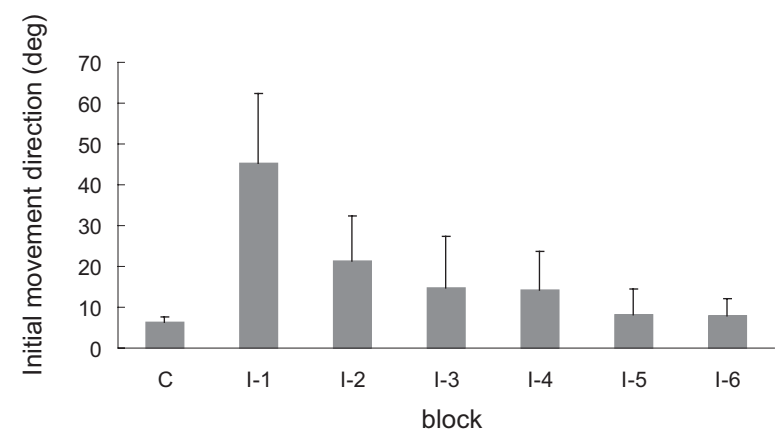

(c)

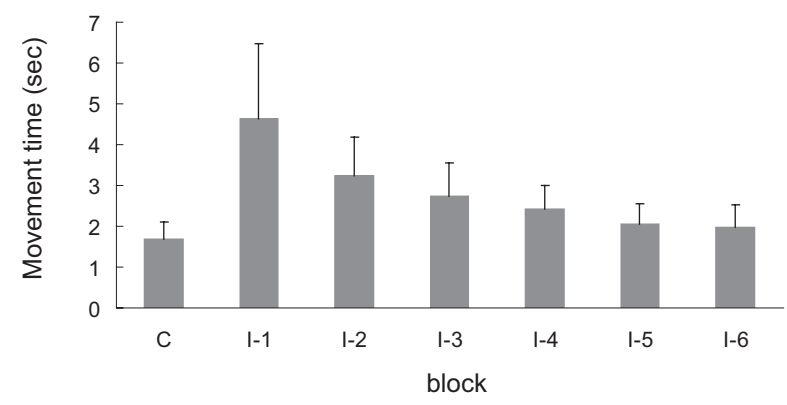

Fig. 6 Behavioral data: (a) Average trajectory error in each block for all the subjects. C, the consistent task; $I-n$, block number of the inconsistent task and (b) Average initial movement direction in each block for all the subjects, and (c) average movement time in each block for all the subjects.

the first block of the inconsistent task, oxy-Hb increased bilaterally in comparison to the consistent task [left, $t(9)=4.65$, $P<0.003$; right, $t(9)=4.92, P<0.002]$ and the increased oxy-Hb gradually decreased with block repetitions. Eventually, there were no differences in changes in oxy-Hb between the

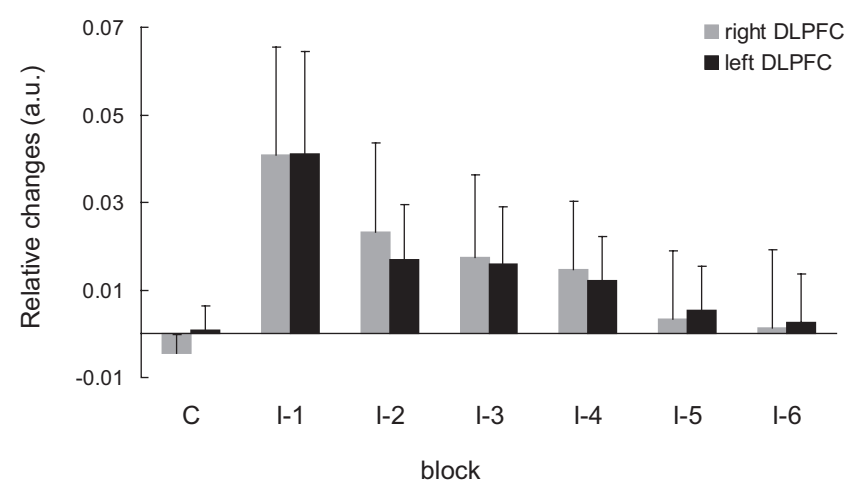

Fig. 7 Variations in mean oxy-Hb changes in the bilateral DLPFC for all the subjects across seven blocks. Changes in oxy-Hb were expressed as an arbitrary unit (a.u.). Vertical bars represent the standard deviation (c, the consistent task; $I-n$, block number of the inconsistent task).

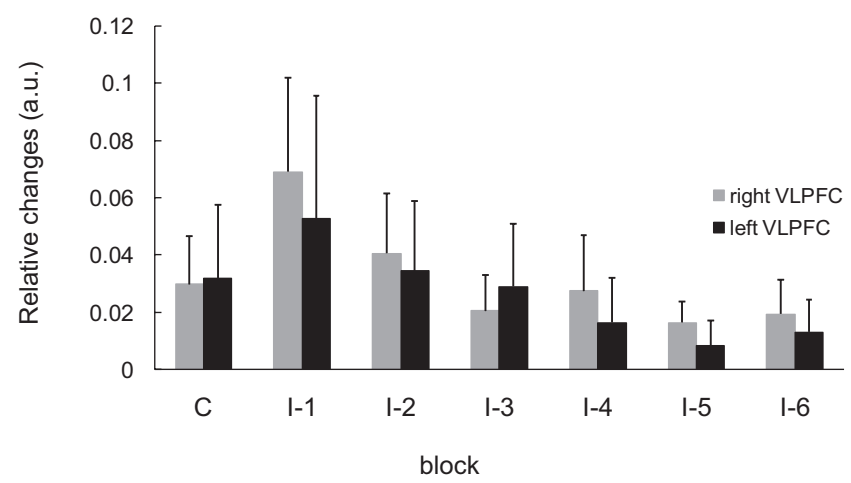

Fig. 8 Variations in mean oxy-Hb changes in the bilateral VLPFC for all the subjects across seven blocks. Changes in oxy-Hb were expressed as an arbitrary unit (a.u.). Vertical bars represent the standard deviation (c, the consistent task; $I-n$, block number of the inconsistent task).

consistent task and the last block of the inconsistent task in both sides of the DLPFC. The degree of oxy-Hb changes in both sides of the DLPFC during the inconsistent task was significantly correlated with task performance [TE: left, $r=0.96$, $P<0.005$; right, $r=0.96, P<0.005$; IMD: left, $r=0.98$, $P<0.0001$; right, $r=0.97, P<0.0005$; MT: left, $r=0.99$, $P<0.00005$; right, $r=0.97, P<0.0005)$.

\subsubsection{VLPFC}

Figure 8 shows changes in the oxy-Hb in each block in the bilateral VFPFC for all subjects. There were significant main effects of the block in oxy-Hb changes between blocks in the right VLPFC $[F(2.15)=22.40, P<0.001]$ but not in the left VLPFC $[F(3.10)=1.55, P=0.223]$, though the relation between oxy$\mathrm{Hb}$ changes and each block in the left VLPFC was similar to that in the right VLPFC. Compared to the "first resting" values, significant increases in oxy-Hb were observed during the consistent task in the right VLPFC $[t(9)=4.56, P<0.01]$. The increase in oxy-Hb in the first block of the inconsistent task was greater than that during the consistent task in the right VLPFC $[t(9)=5.33$, $P<0.001]$. The increased oxy-Hb in the right VLPFC gradually decreased as the block proceeded, which was significantly correlated with task performance [TE, $r=0.94, P<0.005$; IMD, $r=0.91, P<0.005$; MT, $r=0.89, P<0.01]$. There were no significant differences in the degree of changes in oxy- $\mathrm{Hb}$ between the consistent task and the last block of the inconsistent task in the right VLPFC.

\subsection{3 $F P C$}

In the $\mathrm{FPC}$, the direction of change in oxy-Hb during performance of the consistent and inconsistent tasks varied with each block within a single subject. The patterns of changes in oxy- $\mathrm{Hb}$ also varied with each subject. In addition, oxy-Hb tended to increase during the resting periods rather than during performance of the tasks. Grouped data analysis showed that there were no changes in oxy-Hb during the consistent task in comparison to the first resting period [left, $t(11)=-1.74, P=0.142$; right, $t(11)=-1.92, P=0113$ ], and there were no significant differences in oxy-Hb changes between task blocks in both sides of the 


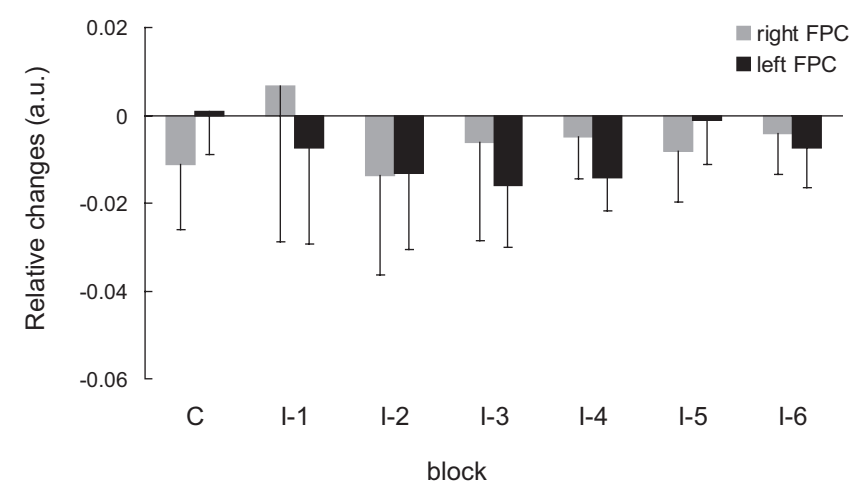

Fig. 9 Variations in mean oxy-Hb changes in the bilateral FPC for all the subjects across seven blocks. Changes in oxy-Hb were expressed as an arbitrary unit (a.u.). Vertical bars represent the standard deviation (c, the consistent task; $I-n$, block number of the inconsistent task).

FPC [left, $F(2.73)=2.40, P=0.116$; right, $F(1.27)=0.521, P$ $=0.539$ ] (Fig. 9).

\section{Discussion}

\subsection{Task performance}

Three behavioral measures (TE, IMD, and MT) were examined to evaluate task performance. Judging from the TE and IMD, which were very small, as anticipated, the motor skill for the consistent task was considered to have been well acquired. In addition, in our pilot study, we examined the three behavioral measures, while six subjects (two males and four females; ranging in ages from 19 to 30 years, mean age 21.5 years), who were not included in the present study, performed five blocks of the consistent tasks without an interblock interval (a total of 60 trials). One-way repeated measures ANOVA showed that there were no differences in the values of all three behavioral measures between the blocks (in preparation). This finding also supported the assumption that the consistent reaching task is well acquired and automatically performed. The task performance in the first block of the inconsistent task was the worst in terms of all three behavioral measures, but it was gradually improved, and in the last block it finally reached the same level as the consistent task. It was thus considered that the subjects adapted themselves to transformed visuomotor relationships in the last block and the motor skill for the inconsistent task was acquired.

\subsection{Changes in Brain Activity}

\subsubsection{DLPFC}

It is now widely accepted that the lateral PFC plays a key role in a wide range of executive functions in the control of cognition and behavior. ${ }^{7,33}$ Petrides ${ }^{34}$ has proposed that there are two executive processing systems within the lateral PFC in working memory: the DLPFC is involved in high-level executive functions, whereas the VLPFC subserves the expression of various first-order executive processes. Our results did not clearly show such hierarchical dichotomy but did reveal the existence of functional segregation of the lateral PFC.

The bilateral DLPFC was activated during the first block of the inconsistent task; whereas, unlike the VLPFC, both sides of the DLPFC were not activated during the consistent task or during the last block of the inconsistent task. This indicated that the DLPFC was bilaterally engaged in higher order cognitive functions, such as mental rotation, sensorimotor transformations, and action planning, which occurred only during the early stage of visuomotor transformation learning. This result was in accordance with the previous neuroimaging studies on the other form of motor learning, where the DLPFC was activated during the early stages of explicit motor learning by trial and error. ${ }^{12,35}$ In these studies, right-left differences in the DLPFC activation were commonly observed; however, which side of the DLPFC was involved depended on the study. Examining the time course of activation during the early, intermediate, and advanced stages of visuomotor sequence learning, Sakai et al. ${ }^{36}$ reported that the initial pronounced activation occurred in the left DLPFC; whereas the prolonged activation of the right DLPFC compared to the left DLPFC was also observed. In contrast, Toni et al., ${ }^{37}$ who examined whole-brain activity throughout the course of motor sequence learning, found that the right DLPFC was activated in the first half of learning but not when the task became overlearned.

The inconsistent task, in which the subjects had to overcome task-irrelevant information, required top-down attentional control and conflict resolution. Recent neuroimaging studies have indicated that the DLPFC is involved in top-down attentional control $^{38,39}$ and conflict resolution. ${ }^{40}$ However, which side of the DLPFC is involved in these cognitive processes remains controversial, as in the case of motor learning. This controversy has been accounted for by differences in control processes engaged by different task paradigms used in the studies. ${ }^{41}$ Thus, it is supposed that the right-left differences in the DLPFC activation during motor learning, including visuomotor transformation learning, also depend on cognitive processes involved in each motor task.

\subsection{2 $V L P F C$}

In the right VLPFC, oxy-Hb was significantly increased during the consistent task compared to the first resting period and increased further in the first block of the inconsistent task; however, the degree of augmentations of oxy-Hb gradually declined with block repetitions and returned to the same level as one during the consistent task. This meant that the right VLPFC was engaged in the performance of reaching tasks not only during visuomotor transformation learning, but also after the motor skills were acquired, which was distinct from the DLPFC.

Compared to the DLPFC, functions of the VLPFC are less understood; although, a number of anatomical studies on nonhuman primates have revealed that the VLPFC is connected with sensory, ${ }^{42-44}$ premotor, ${ }^{45,46}$ and other prefrontal circuitry. ${ }^{47} \mathrm{Re}$ cent neuroimaging studies have demonstrated that the VLPFC is involved in a variety of high cognitive functions, such as motor response control, attention, and working memory. It has been suggested that the VLPFC is engaged in associating visual cues with appropriate actions. ${ }^{48,49}$ Hampshire et al. ${ }^{50}$ have reported that the VLPFC responds only to the target and not to the nontarget during a simple visual attentional task. In the present study, during the consistent and inconsistent tasks, the subjects had to perceive the appearance of a target before moving the cursor from the starting point. This meant that minimal visuomotor 
association was required in both tasks. Thus, one possible explanation for the VLPFC activation during the consistent task is that the VLPFC was engaged in simple visuomotor control (lower order cognitive control) as well as detection of the target.

It has been reported that the VLPFC contributes to the maintenance of spatial information during spatial working memory processing. ${ }^{51,52}$ It is also known that the VLPFC is engaged in the suppression of motor responses to an irrelevant stimulus. ${ }^{53,54}$ Performing the inconsistent task requires maintenance of spatial information about the target and motor response inhibition, both of which are hardly required during performance of the consistent task. Thus, it is conceivable that the VLPFC was activated more by additional loads of the working memory and motor response inhibition, resulting in further increases in oxy-Hb in the VLPFC during the first block of the inconsistent task. These loads were expected to be reduced as learning progressed.

As is shown in Fig. 8, the relationship between changes in oxy-Hb and each task block in the left VLPFC was similar to that in the right VLPFC. However, the statistical analysis indicated that the right VLPFC was exclusively engaged in both lowerand higher order cognitive control of reaching movements. The right VLPFC is thought to play an integral role in inhibiting cognitive control of behavior, ${ }^{55-57}$ whereas several studies have indicated that specific forms of cognitive control are associated with the left VLPFC. ${ }^{49,58}$ It has also been reported that the VLPFC is bilaterally involved in a wide range of higher order cognitive functions. ${ }^{50,59}$ Because of the weak detection power of NIRS, it is hard to know whether the left VLPFC had been activated during consistent and inconsistent reaching tasks.This weak detection power is attributable to the fact that its spatial resolution is poor and NIRS signals at one channel are not independent but can be influenced by those at adjacent channels.

\subsection{3 $F P C$}

In contrast to the VLPFC and the DLPFC, oxy-Hb tended to increase during the resting periods rather than the task periods in the FPC. The group data analysis showed that there were no significant changes in oxy-Hb during either the consistent task or the inconsistent task in the FPC. Thus, it was concluded that the FPC was not directly involved in cognitive control of reaching movements.

The role of the FPC remains to be clearly described, while the activation of this region has frequently been reported by functional neuroimaging studies on complex cognitive tasks. After reviewing articles, Ramnani and Owen ${ }^{60}$ have proposed that the role of the FPC is information processing and information transfer between multiple operations across the supra modal cortex, which combines and coordinates outcomes from multiple cognitive tasks. Christoff and Gabrieli, ${ }^{29}$ who reviewed studies in the domains of reasoning and episodic memory retrieval, concluded that this region was selectively involved in active processing performed on internally generated information. They also described that the FPC might be activated by uncontrolled mental processes occurring during resting conditions, which accounts for the increases in oxy- $\mathrm{Hb}$ during the resting periods in the present study. Thus, it was hypothesized that the FPC was more preferentially engaged in anticipation and evaluation of reaching movements during the resting periods than cognitive processes during the reaching movements.

\section{Conclusions}

In order to elucidate the role of the human PFC in cognitive control of reaching movements, we employed multichannel NIRS to examine brain activity of three subregions (VLPFC, DLPFC, FPC) of the PFC during reaching tasks with a normal visual feedback and with inconsistent visual and proprioceptive feedback. The results indicated that functional segregation for reaching movements exists in the PFC. It was suggested that the DLPFC was engaged in higher order cognitive control of reaching movements, which was required only during the early stage of visuomotor transformation learning. In contrast, the right VLPFC was probably engaged in lower order cognitive control, which was required even after the skills of reaching movements were acquired, as well as higher order cognitive control. It was also hypothesized that the FPC was more preferentially engaged in anticipation and evaluation of reaching movements.

\section{References}

1. M. Kawato, K. Furukawa, and R. Suzuki, "A hierarchical network model for motor control and learning of voluntary movement," Biol. Cybern. 57, 69-185 (1987)

2. A. Battaglia, R. Caminiti, F. Lacquaniti, and M. Zago, "Multiple levels of representation of reaching in the parieto-frontal network," Cereb. Cortex 13, 1009-1021 (2003).

3. D. M. Wolpert and M. Kawato, "Multiple paired forward and inverse models for motor control," Neural Networks 11, 1317-1329 (1998).

4. J. M. Fuster, "Executive frontal functions," Exp. Brain Res. 133, 66-70 (2000).

5. D. Boussaoud and S. P. Wise, "Primate frontal cortex: effects of stimulus and movement," Exp. Brain Res. 95, 28-40 (1993).

6. S. Funahashi, M. Inoue, and K. Kubota, "Delay-period activity in the primate prefrontal cortex encording multiple spatial positions and their order of presentation," Behav. Brain Res. 84, 203-223 (1997).

7. J. Tanji and E. Hoshi, "Role of the lateral prefrontal cortex in executive behavioral control," Physiol. Rev. 88, 37-57 (2008).

8. R. Kawashima, P. E. Roland, and B. T. O'Sullivan, "Functional anatomy of reaching and visuomotor learning: a positron emission tomography study," Cereb. Cortex 5, 111-122 (1995).

9. S. T. Grafton, A. H. Fagg, R. P. Woods, and M. A. Arbib, "Functional anatomy of pointing and grasping in humans," Cereb. Cortex 6, 226-237 (1996).

10. M. S. Beurze, P. F. De Lange, I. Toni, and P. W. Medendorp, "Integration of target and effector information in the human brain during reach planning,"J. Neurophysiol. 97, 188-199 (2007).

11. F. Lacquaniti, D. Perani, E. Guigon, V. Bettinardi, M. Carrozzo, F. Grassi, Y. Rossetti, and F. Fazio, "Visuomotor transformations for reaching to memorized targets: PET study," NeuroImage 5, 129-146 (1997).

12. H. Jenkins, D. J. Brooks, P. D. Nixon, R. S. J. Frackowiak, and R. E. Passingham, "Motor sequence learning: a study with positron emission tomography," J. Neurosci. 14, 3775-3790 (1994).

13. M. Jueptner, K. M. Stephan, C. D. Frith, D. J. Brooks, R. S. J. Frackowiak, and R. E. Passingham, "Anatomy of motor learning. I. frontal cortex and attention to action," J. Neurophysiol. 77, 1313-1324 (1997).

14. M. Redding and B. Wallace, "Generalization of prism adaptation," J. Exp. Psychol. 32, 1006-1022 (2006).

15. A. Roby-Brami and Y. Burnod, "Learning a new visuomotor transformation: error correction and generalization," Cogn. Brain Res. 2 , 229-242 (1995)

16. Z. Ghahramani and D. M. Wolpert, "Modular decomposition in visuomotor learning," Nature 386, 392-395 (1997).

17. M. Clower, J. M. Hoffmanm, J. R. Votaw, T. L. Faber, R. P. Woods, and G. E. Alexander, "Role of posterior parietal cortex in the recalibration of visually guided reaching," Nature 383, 618-621 (1996).

18. X. Graydon, K. J. Friston, C. G. Thomas, V. B. Brooks, and R. S. Menon, "Learning-related fMRI activation associated with a rotational visuo-motor transformation," Cogn. Brain Res. 22, 373-383 (2005). 
19. J. Luautè, S. Schwartz, Y. Rossetti, M. Spiridon, G. Rode, D. Boisson, and P. Vuilleumier, "Dynamic changes in brain activity during prism adaptation," J. Neurosci. 29, 169-178 (2009).

20. R. D. Seider, D. C. Noll, and P. Chintalapati, "Bilateral basal ganglia activation associated with sensorimotor adaptation," Exp. Brain Res. 175, 544-555 (2006).

21. M. Girgenrath, O. Bock, and R. J. Seitz, "An fMRI study of brain activation in a visual adaptation task: activation limited to sensory guidance," Exp. Brain Res. 184, 561-569 (2008).

22. E. Hoshi, K. Shima, and J. Tanji, "Neuronal activity in the primate prefrontal cortex in the process of motor selection based on two behavioral rules," J. Neurophysiol. 83, 2355-2373 (2000).

23. Y. Hoshi and M. Tamura, "Detection of dynamic changes in cerebral oxygenation coupled to neuronal function during mental work in man," Neurosci. Lett., 150 5-8 (1993).

24. T. Kato, A. Kamei, S. Takashima, and T. Ozaki, "Human visual cortical function during photic stimulation monitoring by means of near-infrared spectroscopy," J. Cereb. Blood Flow Metab. 13, 516-520 (1993).

25. A. Villringer, J. Planck, C. Hock, L. Schleinkofer, and U. Dirnagl, "Near-infrared spectroscopy (NIRS): a new tool to study hemodynamic changes during activation of brain function in human adults," Neurosci. Lett. 154, 101-104 (1993).

26. F. Okada, Y. Tokumitsu, Y. Hoshi, and M. Tamura, "Gender- and handedness-related differences of forebrain oxygenation and hemodynamics," Brain Res. 601, 337-342 (1993).

27. Y. Fukuji, T. Bise, H. Hokama, K. Hiramatsu, and C. Ogura, "Changes of cerebral blood volume in frontal region during mirror drawing task measured by two channel NIRS in schizophrenics," Int. Congr. Ser. 1232, 667-671 (2002).

28. S. Shimada, K. Hiraki, G. Matsuda, and I. Oda, "Decrease in prefrontal hemoglobin oxygenation during reaching tasks with delayed visual feedback: a near-infrared spectroscopy study," Cogn. Brain Res. 20, 480-490 (2004)

29. K. Christoff and J. D. E. Gabrieli, "The frontopolar cortex and human cognition: evidence for a rostrocaudal hierarchical organization within the human prefrontal cortex," Psychobiology 28, 168-186 (2001).

30. T. Delpy, M. Cope, P. van der Zee, S. R. Arridge, S. Wray, and J. Wyatt, "Estimation of optical pathlength through tissue from direct time of flight measurement," Phys. Med. Biol. 33, 1433-1442 (1988).

31. Y. Hoshi, O. Hazeki, Y. Kakihana, and M. Tamura, "Redox behavior of cytochrome oxidase in the rat brain measured by near-infrared spectroscopy," J. Appl. Physiol. 83, 1842-1845 (1997).

32. Y. Hoshi, N. Kobayashi, and M. Tamura, "Interpretation of near-infrared spectroscopy signals,” J. Appl. Physiol. 90, 1657-1662 (2001).

33. M. Petrides, "Lateral prefrontal cortex: architectonic and functional organization,” Phil. Trans. R. Soc. B 360, 781-795 (2005).

34. M. Petrides, "Frontal lobes and working memory: evidence from investigations of the effects of cortical excisions in non-human primates," in Handbook of Neuropsychology. F. Boller and J. Grafmann, Eds., pp. 59-84, Elsevier, Amsterdam (1994).

35. U. Halsband and R. K. Lange, "Motor learning in man: a review of functional and clinical studies," J. Physiol. Paris. 99, 414-424 (2006).

36. K. Sakai, O. Hikosaka, S. Miyauchi, R. Takino, Y. Sasaki, and P. Benno, "Transition of brain activation from frontal to parietal areas in visuomotor sequence learning," J. Neurosci. 18, 1827-1840 (1998).

37. I. Toni, M. Krams, R. Turner, and R. E. Passingham, "The time course of changes during motor sequence learning: a whole brain fMRI study," Neurolmage 8, 50-61 (1988).

38. A. W. MacDonald III, J. D. Cohen, V. A. Stenger, and C. S. Carte, "Dissociating the role of the dorsolateral prefrontal and anterior cingulated cortex in cognitive control," Science 288, 1835-1838 (2000).

39. M. P. Milham, M. T. Banich, and V. Barad, "Competition for priority in processing increases prefrontal cortex's involvement in top-down control: an event-related fMRI study of the stroop task," Cogn. Brain Res. 17, 212-222 (2003).

40. C. Liston, S. Matalon, T. A, Hare, M. C. Davidson, and B. J. Casey, "Anterior cingulated and posterior parietal cortices are sensitive to dissociable forms of conflict in a task-switching paradigm," Neuron 50, 643-653 (2006)

41. J. G. Kerns, J. D. Cohen, A. W. MacDonald III, R. Y. Cho, V. A. Stenger, and C. S. Carter, "Anterior cingulated conflict monitoring and adjustments in control," Science 303, 1023-1026 (2004)

42. E. G. Jones and T. P. S. Powell, "An anatomical study of cognitive sensory pathways within the cerebral cortex of the monkey," Brain 93, 793-820 (1970).

43. G. Ungerleider, D. Gaffan, and V. S. Pelak, "Projections from inferior temporal cortex to prefrontal cortex via the uncinate fascicle in rhesus monkeys," Exp. Brain Res. 76, 473-484 (1989).

44. C. Cavada and P. S. Goldman-Rakic, "Posterior parietal cortex in rhesus monkey: II. evidence for segregated corticocortical networks linking sensory and limbic areas with the frontal lobe," J. Comp. Neurol. 287, 422-445 (1989)

45. S. Miyachi, X. Lu, S. Inoue, T. Iwasaki, S. Koike, A. Nambu, and M. Takada, "Organization of multisynaptic inputs from prefrontal cortex to primary motor cortex as revealed by retrograde transneuronal transport of rabies virus," J. Neurosci. 25, 2547-2556 (2005).

46. M. Sakagami and X. Pan, "Functional role of the ventrolatral prefrontal cortex in decision making," Curr. Opin. Neurobiol. 17, 228-233 (2007).

47. M. Petrides and D. N. Pandya, "Comparative cytoarchitectonic analysis of the human and the macaque ventrolateral prefrontal cortex and corticocortical connection patterns in the monkey," Eur. J. Neurosci. 16, 291-310 (2001).

48. R. E. Passingham, I. Toni, and M. F. S. Rushworth, "Specialisation within the prefrontal cortex: the ventral prefrontal cortex and associative learning," Exp. Brain Res. 133, 103-113 (2000).

49. S. A. Bunge, I. Kahn, J. D. Wallis, E. K. Miller, and A. D. Wagner, "Neural circuits subserving the retrieval and maintenance of abstract rules," J. Neurophysiol. 90, 3419-1428 (2003).

50. A. Hampshire, J. Duncan, and A. M. Owen, "Selective tuning of the blood oxygenation level-dependent response during simple target detection dissociates human frontoparietal subregions," J. Neurosci. 27, 6219-6223 (2007).

51. A. M. Owen, A. C. Evans, and M. Petrides, "Evidence for a two-stage model of spatial working memory processing within the lateral frontal cortex: a positron emission tomography study," Cereb. Cortex $\mathbf{6}, 31-38$ (1996).

52. M. Toepper, H. Gebhardt, T. Beblo, C. Thomas, M. Driessen, M. Bischoff, C. R. Blecker, D. Vaitl, and G. Sammer, "Functional correlates of distractor suppression during spatial working memory encoding," Neuroscience 165, 1244-1253 (2010).

53. P. F. Liddle, K. A. Kiehl, and A. M. Smith, "Event-related fMRI study of response inhibition," Hum. Brain Mapp. 12, 100-109 (2001)

54. H. C. Leung and W. Cai, "Common and differential ventrolateral prefrontal activity during inhibition of hand and eye movements," J. Neurosci. 27, 9893-9900 (2007).

55. S. Konishi, K. Nakajima, I. Uchida, H. Kikyo, M. Kameyama, and Y. Miyashita, "Common inhibitory mechanism in humam inferior prefrontal cortex revealed by event-related functional MRI," Brain 122, 981-991 (1999).

56. R. Aron, T. W. Robbins, and R. A. Poldrack, "Inhibition and the right inferior frontal cortex," Trends Cog. Sci. 8, 170-177 (2004).

57. B. J. Levy and A. D. Wagner, "Cognitive control and right ventrolateral prefrontal cortex: reflexive reorienting, motor inhibition, and action updating," Ann. N. Y. Acad. Sci., 1224 40-62 (2011).

58. D. Badre and A. D. Wagner, "Left ventrolateral prefrontal cortex and the cognitive control of memory," Neuropsychologia 45, 2883-2901 (2007).

59. R. E. Passingham and I. Toni, "Contrasting the dorsal and ventral visual systems: guidance of movement versus decision making," Neuroimage 14, S125-S131 (2001).

60. N. Ramnani and A. M. Owen, "Anterior prefrontal cortex: Insights into function from anatomy and neuroimaging," Nat. Rev. 5, 184-194 (2004). 